\title{
Microbial communities associated with the invasive Hawaiian sponge Mycale armata
}

\author{
Guangyi Wang, Sang-Hwal Yoon and Emilie Lefait \\ Department of Oceanography, University of Hawaii at Manoa, Honolulu, HI, USA
}

\begin{abstract}
Microbial symbionts are fundamentally important to their host ecology, but microbial communities of invasive marine species remain largely unexplored. Clone libraries and Denaturing gradient gel electrophoresis analyses revealed diverse microbial phylotypes in the invasive marine sponge Mycale armata. Phylotypes were related to eight phyla: Proteobacteria, Actinobacteria, Bacteroidetes, Cyanobacteria, Acidobacteria, Chloroflexi, Crenarchaeota and Firmicutes, with predominant alphaproteobacterial sequences $(>58 \%)$. Three Bacterial Phylotype Groups (BPG1associated only with sequence from marine sponges; BPG2-associated with sponges and other marine organisms and BPG3-potential new phylotypes) were identified in $M$. armata. The operational taxonomic units (OTU) of cluster BPG2-B, belonging to Rhodobacteraceae, are dominant sequences of two clone libraries of $M$. armata, but constitute only a small fraction of sequences from the non-invasive sponge Dysidea sp. Six OTUs from $M$. armata were potential new phylotypes because of their low sequence identity with their reference sequences. Our results suggest that $M$. armata harbors both sponge-specific phylotypes and bacterial phylotypes from other marine organisms.

The ISME Journal (2009) 3, 374-377; doi:10.1038/ismej.2008.107; published online 6 November 2008 Subject Category: microbial ecology and functional diversity of natural habitats

Keywords: sponge-microbe symbiosis; invasive marine sponges; microbial diversity; Mycale armata
\end{abstract}

Marine invasive species pose a serious threat to the world's oceans. Symbiotic microbes can dramatically impact ecosystem function by changing the phenotype of their hosts (Gerardo et al., 2006). However, microbial communities within invasive marine species remain relatively unknown. The marine sponge Mycale armata (family Mycalidae) is a recent, unintentionally introduced sponge species, becoming invasive and the most abundant sponge species in the Hawaiian reef ecosystem. In Kaneohe Bay (Oahu, Hawaii), this species overgrows and kills native coral lagoon-patch reef communities (Supplementary Figure 1). To reveal microbial features of $M$. armata, molecular methods were applied to investigate its microbial communities.

Sponge samples were collected along the shores of Coconut Island in August 2004 and May 2005, situated within Kaneohe Bay and surrounded by 64 acres of coral reef. Samples of sea water and sponges (invasive and non-invasive) were collected and processed according to the method of Gao et al. (2008). Total genomic DNA was used as a PCR template for the amplification of $16 \mathrm{~S}$ rRNA genes for

Correspondence: G Wang, Department of Oceanography, University of Hawaii at Manoa, 1000 Pope Road, MSB205, Honolulu, HI 96822, USA.

E-mail: guangyi@hawaii.edu

Received 1 September 2008; revised 2 October 2008; accepted 8 October 2008; published online 6 November 2008 library construction and denaturing gradient gel electrophoresis analysis (Gao et al., 2008; Zhu et al., 2008). Band patterns of microbial communities varied in different sponge species collected at the same time, but bands were similar in samples of the same sponge species collected at different times (Figure 1). Seven major bands were consistently present in samples of $M$. armata collected in both years. Two bands (1 and 2), which were common to M. armata and other non-invasive sponges (Dysidea sp., Gelliodes fibrosa, Tedania sp. and Scopalina sp.), and two other, unique bands (3 and 4) were detected in the samples of $M$. armata collected in both years. The denaturing gradient gel electrophoresis band patterns suggested that M. armata contained microbial phylotypes present in the other Hawaiian sponges, but also harbored unique microbial phylotypes and these phylotypes seemed to have little temporal changes.

To reveal microbial composition in M. armata, four clone libraries were constructed from the samples of M. armata (A and B for samples collected in August 2004 and May 2005, respectively), Dysidea sp. (C) and sea water (D). Clone sequences were de-replicated first and then grouped into the same operational taxonomic unit (OTU) using a percent sequence identity of greater than $97 \%$. The percentage coverage and diversity of the four libraries are summarized in Supplementary Table 1. Analysis of clone sequences identified 24 and 19 


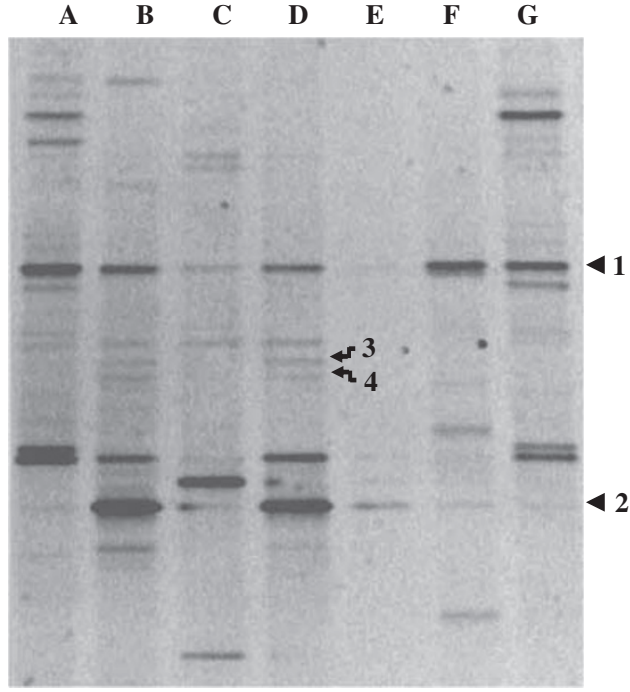

Figure 1 Denaturing gradient gel electrophoresis (DGGE) banding profiles of bacterial 16S rRNA genes PCR-amplified from total DNA extracted from sponges using the primers GC-BAC341 and BAC521. Lanes A and G, Dysidea sp.; lane C, Gelliodes fibrosa; lanes B and D, Mycale armata; lane E, Tedania sp. and lane F, Scopalina sp. Lanes D-G, samples collected at Kaneohe bay in August 2004. Lanes A-C, samples collected in May 2005 from the same location. PCR products were concentrated and prepared as described by Gao et al. (2008).

OTUs from $M$. armata libraries A and B, respectively. Additional 27 and 15 OTUs were identified from clone libraries of Dysidea sp. (C) and sea water (D), respectively. Sequences of $16 \mathrm{~S}$ rRNA gene clones from $M$. armata libraries were related to members of Proteobacteria (classes Alpha-, Beta-, Delta- and Gammaproteobacteria), Bacteroidetes, Actinobacteria, Cyanobacteria, Firmicutes, Chloroflexi, Acidobacteria and Crenarchaeota (Figure 2; Supplementary Figures 2 and 3). Alphaproteobacteria were a dominant microbial group, comprising $61.3 \%$ and $58.0 \%$ of clone sequences from libraries A and B, respectively. Although alphaproteobacterial sequences have been observed in 16S rRNA gene libraries constructed from over 12 marine sponges collected from several ocean provinces (Hentschel et al., 2006; Taylor et al., 2007; Thiel et al., 2007a, b; Zhu et al., 2008), this is only the second report of alphaproteobacterial dominance in the 16S rRNA clone library, after the report on Halichondria panicea (Wichels et al., 2006). The three classes (Alpha-, Gamma- and Deltaproteobacteria) and three phyla (Actinobacteria, Cyanobacteria and Bacteroidetes) were found in both $M$. armata clone libraries (A and B). Sequences of chloroplasts and the phylum Verrucomicrobia were found exclusively in Dysidea sp. whereas members of Actinobacteria, Crenarchaeota and Deltaproteobacteria were present only in one (A or B) library of M. armata (Figure 2). Fewer phyla were identified in the seawater library, but unclassified bacterial and euryarchaeote sequences (Supplementary Figure 2) were present only in the seawater library.
Three Bacterial Phylotype Groups (BPGs) were identified from OTUs derived from $M$. armata and Dysidea sp. (Figure 2). The first group (BPG1) included 10 OTUs affiliated only with spongederived 16S rRNA bacterial sequences. Of this group, three OTUs of cluster BPG1-D were members of Gammaproteobacteria and found in the samples of $M$. armata collected in both years. Three OTUs of clade BPG1-B were closely clustered with betaproteobacterial sequences identified in Mediterranean sponge Tethya aurantium (Thiel et al., 2007b) and the Antarctic sponges Latrunculia apicalis and $M$. acerata (Webster et al., 2004). The OTUs of two clusters BPG1-C and BPG1-D (Figure 2) were phylogenetically related to gammaproteobacterial sequences from the Hawaiian sponges Suberites zeteki (Zhu et al., 2008) and the Great Barrier Reef sponge Rhopaloeides odorabile (Webster et al., 2001), respectively.

The second group (BPG2) contained 14 OTUs related to $16 \mathrm{~S}$ rRNA bacterial sequences from both sponges and other marine organisms, such as corals and squids. Seven OTUs of cluster BPG2-B belonged to Alphaproteobacteria and were present in all samples of M. armata and Dysidea sp. (Figure 2). These OTUs were predominant among clone sequences from two M. armata libraries, but contributed only to a small fraction of clone sequences from Dysidea sp. The OTUs of clusters BPG2-C and BPG2E were members of Alpha- and Gammaproteobacteria, respectively, and were found in the samples of $M$. armata collected in both years (Figure 2). Interestingly, two OTUs (MA17 and MB73) of cluster BPG2C were closely related to Pseudovibrio denitrificans isolated from sea water in Taiwan (Shieh et al., 2004 ) and to the $P$. denitrificans-like clone sequences from 11 marine sponges (Enticknap et al., 2006; Hentschel et al., 2006; Taylor et al., 2007) with $98 \%$ and $99 \%$ sequence identity, respectively, in $16 \mathrm{~S}$ rRNA gene sequences. Because of potential function of the $P$. denitrificans-like phylotypes in host nitrogen metabolism (Webster and Hill, 2001; Enticknap et al., 2006), these OTUs are a promising target for future exploration of their physiological function in $M$. armata. The last group (BPG3), including six OTUs, comprised potentially new bacterial phylotypes because they branched into new clusters, without immediate phylogenetic neighbors (for example, MA47 and MA43). The remaining OTUs were the nonspecific sequences because of their phylogenetic affiliation with $16 \mathrm{~S}$ rRNA gene sequences from sea water, sediments and other environments (for example, MB12 and DC31).

Finally, most OTUs of Bacteroidetes, Acidobacteria, Firmicutes, Actinobacteria, Chloroflexi and Crenarchaeota from $M$. armata and Dysidea sp. displayed close phylogenetic affiliations with sequences derived from sea water or sediments, instead of from marine sponges (Supplementary Figure 2). None of the cyanobacterial sequences from M. armata and Dysidea sp. were related to the 


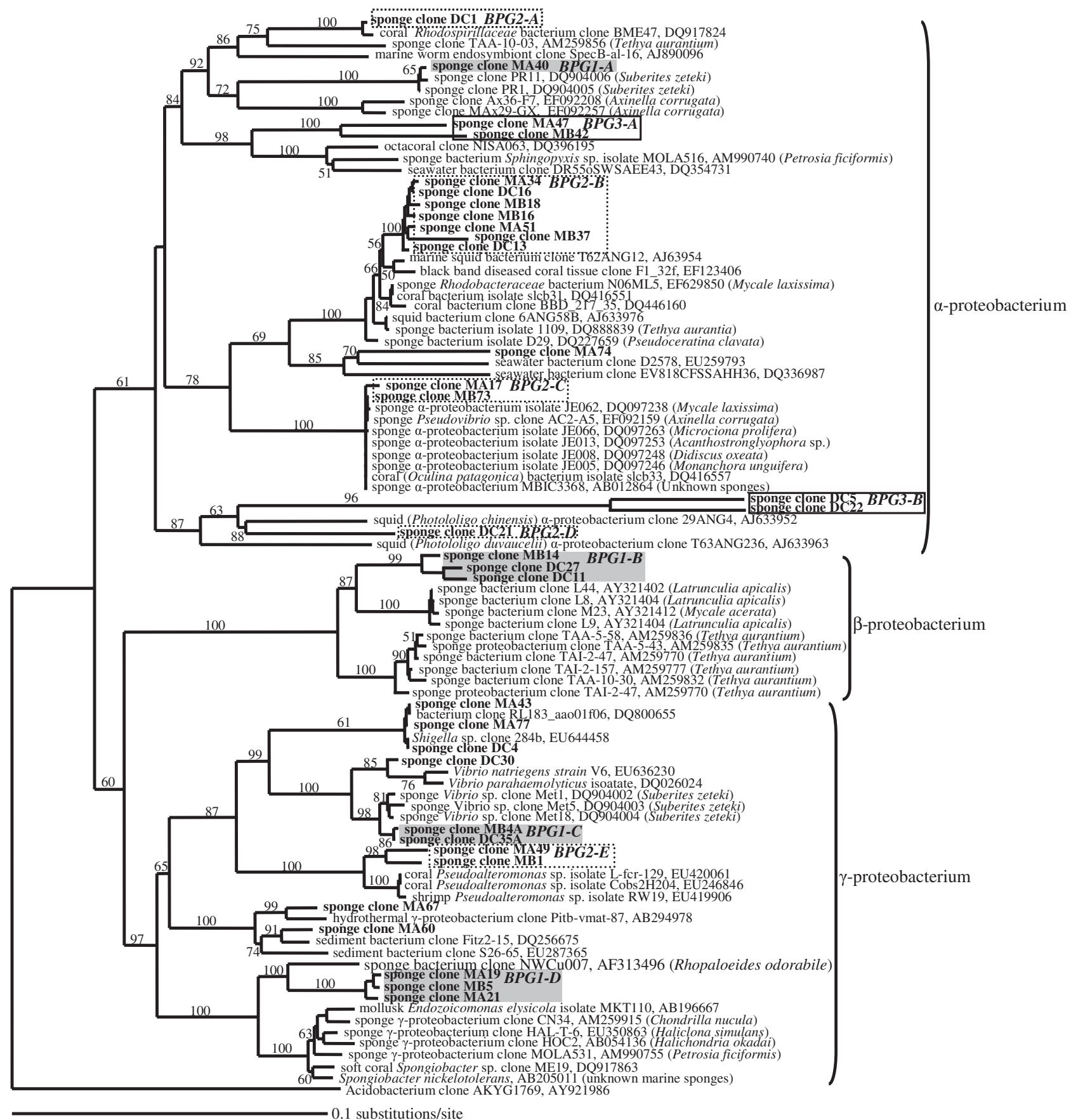

Figure 2 Neighbor-joining phylogenetic tree of proteobacterial 16S rRNA gene sequences derived from the Hawaiian sponges Mycale armata and Dysidea sp. Clone sequences from the 16S rRNA clone libraries A and B for M. armata, library C for Dysidea sp., and library D for sea water are designated with the prefix MA, MB, DC and SW, respectively, and shown in bold. Libraries A, C, and D were constructed from the samples of M. armata, Dysidea sp. and sea water, respectively, were collected in August 2004; library B constructed from the samples of M. armata were collected in May 2005. For matched sequences derived from sponges, the sponge species name is given in parentheses following the sequence accession number. Shaded sequences are OTUs belonging to one of three bacterial phylotype groups (BPG1, BPG2 and BPG3). Numbers above branches indicate bootstrap values of neighbor-joining analysis (>50\%) from 1000 replicates.

sponge-specific cyanobacterium Candidatus Synechococcus spongiarum (Usher et al., 2004) (Supplementary Figure 3).

Invertebrate endosymbioses have major ecological impacts both on habitat range and on the ecosystems of which they are a part. Symbioses with autotrophic symbionts have allowed several marine species, such as scleractinian corals, pogonophoran and lucinid bivalves, to lead partially or exclusively autotrophic lives in tropical reefs, sulfur-rich habitats and other marine environments (Saffo, 1992). Sponge symbionts are thought to benefit their hosts in many ways (Wang, 2006; Taylor et al., 2007). This study revealed the presence of potentially new 
bacterial phylotypes (for example, MA47 and MB42; Figure 2) and sponge-symbiont phylotypes (for example, MA17 and MB73; Figure 2) in sponge M. armata. It represents the first report of microbial communities of this invasive marine species. As microbial symbionts have been shown to play an essential role in the invasiveness of their terrestrial hosts (Clay et al., 2005; Lafay and Burdon, 2006; Parker et al., 2007), further study of these OTUs may lead to the understanding of the invasive nature of M. armata.

\section{Acknowledgements}

We thank D Henderson for her suggestions in greatly improving this paper. We thank J Mathews and F Qi for sponge sample collection. This study was funded by National Oceanic and Atmospheric Administration (NOAA) grant NA04OAR4600196. The views expressed herein are those of the authors and do not necessarily reflect the views of NOAA or any of its subagencies.

\section{References}

Clay K, Holah J, Rudgers JA. (2005). Herbivores cause a rapid increase in hereditary symbiosis and alter plant community composition. Proc Natl Acad Sci USA 102: 12465-12470.

Enticknap JJ, Kelly M, Peraud O, Hill RT. (2006). Characterization of a culturable alphaproteobacterial symbiont common to many marine sponges and evidence for vertical transmission via sponge larvae. Appl Environ Microbiol 72: 3724-3732.

Gao Z, Li BL, Zheng CC, Wang G. (2008). Molecular detection of fungal communities in the Hawaiian marine sponges Suberites zeteki and Mycale armata. Appl Environ Microbiol 74: 6091-6101.

Gerardo NM, Mueller UG, Currie CR. (2006). Complex host-pathogen coevolution in the Apterostigma fungusgrowing antimicrobe symbiosis. BMC Evol Biol 6: 88.

Hentschel U, Usher KM, Taylor MW. (2006). Marine sponges as microbial fermenters. FEMS Microbiol Ecol 55: $167-177$.

Lafay B, Burdon JJ. (2006). Molecular diversity of rhizobia nodulating the invasive legume Cytisus scoparius in Australia. J Appl Microbiol 100: 1228-1238.

Parker MA, Wortz AK, Paynter Q. (2007). Nodule symbiosis of invasive Mimosa pigra in Australia and in ancestral habitats: a comparative analysis. Biol Invasions 9: 127-138.

Saffo MB. (1992). The impact of symbiosis on invertebrate physiology, ecology, and evolution: invertebrates in endosymbiotic associations. Am Zool 32: 557-565.

Shieh WY, Lin YT, Jean WD. (2004). Pseudovibrio denitrificans gen. nov., sp nov., a marine, facultatively anaerobic, fermentative bacterium capable of denitrification. Int $J$ syst Evol Microbiol 54: 2307-2312.

Taylor MW, Radax R, Steger D, Wagner M. (2007). Spongeassociated microorganisms: evolution, ecology, and biotechnological potential. Microb Mol Biol Rev 71: 295-347.

Thiel V, Leininger S, Schmaljohann R, Bruemmer F, Imhoff JF. (2007a). Sponge-specific bacterial associations of the Mediterranean sponge Chondrilla nucula (Demospongiae, Tetractinomorpha). Microb Ecol 54: 101-111.

Thiel V, Neulinger SC, Staufenberger T, Schmaljohann R, Imhoff JF. (2007b). Spatial distribution of spongeassociated bacteria in the Mediterranean sponge Tethya aurantium. FEMS Microbiol Ecol 59: 47-63.

Usher KM, Toze S, Fromont J, Kuo J, Sutton DC. (2004). A new species of cyanobacterial symbiont from the marine sponge Chondrilla nucula. Symbiosis 36: 183-192.

Wang G. (2006). Diversity and biotechnological potential of the sponge-associated microbial consortia. J Ind Microbiol Biotechnol 33: 545-551.

Webster NS, Hill RT. (2001). The culturable microbial community of the Great Barrier Reef sponge Rhopaloeides odorabile is dominated by an alphaproteobacterium. Mar Biol 138: 843-851.

Webster NS, Negri AP, Munro M, Battershill CN. (2004). Diverse microbial communities inhabit Antarctic sponges. Environ Microbiol 6: 288-300.

Webster NS, Webb RI, Ridd MJ, Hill RT, Negri AP. (2001). The effects of copper on the microbial community of a coral reef sponge. Environ Microbiol 3: 19-31.

Wichels A, Wuertz S, Doepke H, Schuett C, Gerdts G. (2006). Bacterial diversity in the breadcrumb sponge Halichondria panicea (Pallas). FEMS Microbiol Ecol 56: 102-118.

Zhu P, Li Q, Wang G. (2008). Unique microbial signatures of the alien Hawaiian marine sponge Suberites zeteki. Microb Ecol 55: 406-414.

Supplementary Information accompanies the paper on The ISME Journal website (http://www.nature.com/ismej) 